CIENCIA Y SOCIEDAD

Volumen XV, Número 3

Julio - Septiembre 1990

\title{
LA TASA DE INTERES Y EL FINANCIAMIENTO A LA MICROEMPRESA Y EL SECTOR INFORMAL EN LA REPUBLICA DOMINICANA
}

ROLANDO REYES ${ }^{*}$

\begin{abstract}
Resumen
En las instituciones dedicadas a la promoción de la microempresa y el sector informal aparece la contradicción entre rentabilidad social y financiera como estrategias de trabajo. Se propone un modelo de rentabilidad compuesta que permitiría eliminar dicha contradiccion.

Palabras claves: Microempresas, sector informal, tasa de interés.
\end{abstract}

En República Dominicana, al igual que otros países de América Latina, una de las formas más importantes de promoción del sector informal y la microempresa es el financiamiento. Dos de los aspectos que se han considerado con más atención son la tasa de interés y las fuentes de financiamiento.

Las presentes notas plantean algunas consideraciones preliminares en torno a estos problemas. Se pretende constituir un planteamiento inicial para la amplia discusión que amerita la complejidad e importancia del tema.

* Escuela de Economía, INTEC. 


\section{El problema de la tasa de interés}

El tema que se aborda en este trabajo ha sido muy debatido y existen diferentes posiciones en torno al mismo. Estas posiciones pueden ser divididas en dos grandes grupos: los que están de acuerdo con que la tasa de interés de los préstamos dirigidos a las microempresas y el sector informal se fije de acuerdo a criterios que tomen en cuenta ciertas características básicas del sector, y los que están de acuerdo con que dicha tasa sea la "tasa de interés del mercado".

La posición a favor de una tasa de interés no necesariamente determinada en base a las condiciones del mercado tiene como principales argumentos los siguientes: a) La microempresa no tiene acceso a los mercados financieros tradicionales, entre otras razones porque no reúne las condiciones de riesgos adecuadas según los esquemas de este tipo de institución financiera. b) La microempresa no tiene capacidad financiera para operar con financiamientos a la tasa de interés del mercado tradicional, dado que normalmente éstas no reúnen las condiciones de rentabilidad y liquidez que se requieren para cumplir con las condiciones de estos préstamos. c) La microempresa y el sector informal, en tanto reúne una serie de condiciones socialmente deseables, requiere de ciertas formas de promoción entre las cuales la más importante es el financiamiento a bajo costo. ${ }^{1}$

La posición a favor de una tasa de interés del mercado se justifica por los siguientes argumentos: a) Ante la imposibilidad de acceder a los mercados financieros tradicionales, los "empresarios" informales recurren a préstamos de otros mercados en donde la tasa de interés tiene un costo más elevado. b) La tasa de interés del mercado refleja el verdadero costo de oportunidad del dinero y en consecuencia los recursos que se asignan a través de este precio se dirigen a las actividades más eficientes. Por tanto, se desprende de lo anterior que la tasa de interés debe ser libre, y es necesario permitir que la oferta y la demanda determinen su valor en el mercado financiero libre de interferencias. ${ }^{2}$

El planteamiento de la tasa de interés del mercado supone que el mercado financiero es de competencia perfecta y que en consecuencia, existe una sola tasa de interés para todas las unidades productivas y para todos los demandantes de financiamiento. Sin embargo, existen imperfecciones tanto por el lado de la demanda como por el lado de la oferta, las cuales determinan que no exista una sola tasa de interés. ${ }^{3}$ Desde el punto de vista de las pequeñas unidades productivas del sector informal, 
sus condiciones de estabilidad, rentabilidad y escala de producción determinan que su demanda de financiamiento se destine más frecuentemente a capital de trabajo, ${ }^{4}$ y lo que es más importante, el monto del financiamiento demandado es muy pequeño en relación al financiamiento demandado por las empresas grandes del sector moderno. $\mathrm{Si}$ el costo del financiamiento está afectado por su monto, y dado que en el mercado de bienes una gran parte de la producción informal compite con la producción de las empresas modernas, entonces la tasa de interés en el financiamiento al sector informal debería por lo menos ser igual a la tasa de interés cobrada en los préstamos grandes destinados a las empresas del sector moderno, si al menos se quiere no penalizar a las pequeñas unidades de producción mediante el costo de financiamiento.

\section{La rentabilidad social y el problema de la institución de financiamiento}

Los argumentos en favor y en contra de la tasa de interés del mercado pueden plantearse como la contradicción existente entre el criterio de la rentabilidad social y la rentabilidad financiera. En un caso se argumenta a favor de que la tasa de interés se determine en función de la rentabilidad social, mientras que en el otro se demanda que se determine en función de la rentabilidad financiera.

Por rentabilidad social se entiende la medida en que el sector informal y la microempresa contribuye eficientemente al logro de ciertos objetivos socialmente deseables, tales como creación de empleos. Por rentabilidad financiera se entiende básicamente capacidad de pago y liquidez, medida mediante la tasa de retorno sobre la inversión que determinan los flujos de fondos calculados usando los precios de mercados.

En el país existen muchas instituciones que se dedican a la promoción de la microempresa y el sector informal a través del financiamiento. Es en estas instituciones en donde la contradicción entre el objetivo de la rentabilidad social y la rentabilidad financiera se refleja con más intensidad. Si las carteras de estas instituciones son administradas con el solo criterio de la rentabilidad social es probable que éstas disminuyan su valor real a un ritmo acelerado, viéndose condenada a desaparecer al cabo de cierto tiempo. Pero si asignan sus recursos solamente bajo el criterio de la rentabilidad financiera, es muy probable 
que los objetivos de los beneficios sociales que se plantean estas instituciones no sean alcanzados.

\section{Una alternativa conciliadora}

Es posible demostrar que los objetivos antes señalados no necesariamente son mutuamente excluyentes, y que se pueden plantear criterios alternativos al problema de la determinación de la tasa de interés en el financiamiento a las pequeñas unidades productivas del sector informal. Un criterio consiste en fijar la tasa de interés en función de ambos tipos de rentabilidad, comparando esta rentabilidad compuesta con el costo del capital de la institución que patrocina el programa de financiamiento.

La tasa de interés será una función negativa de la rentabilidad social, pero positiva de la rentabilidad financiera, estableciendo ponderaciones para cada tipo de rentabilidad, dependiendo del valor de la rentabilidad financiera. La ponderación depende de la rentabilidad financiera, ya que ésta es la que mide la capacidad del proyecto para soportar determinados niveles de gastos financieros. Pero esto no quiere decir que la tasa de interés se determine en función de ésta exclusivamente como se verá a continuación.

En principio, pueden darse dos casos. El primero es que la rentabilidad financiera sea menor que el costo del capital de la institución, y el segundo es lo inverso, es decir, que la rentabilidad financiera sea mayor que el costo del capital. A continuación se analizará cada caso por separado, y para ello se utilizarán las abreviaciones RF para hacer referencia a la rentabilidad financiera, $R S$ para la rentabilidad social, y $\mathrm{K}$ para el costo del capital.

En el primer caso, si la rentabilidad financiera es menor que el costo de capital, se puede expresar dicha rentabilidad como un coeficiente en relación a dicho costo:

$$
\mathrm{a}=\mathrm{RF} / \mathrm{K}
$$

que como es lógico, será menor que la unidad. Dado que la rentabilidad financiera no es el único criterio y por tanto el proyecto no es rechazado por esa condición, se hace necesario medir la rentabilidad compuesta tomando en cuenta también la rentabilidad social. Dicha rentabilidad compuesta puede definirse como $\mathrm{R}$ en la siguiente expresión:

$$
\mathrm{R}=\mathrm{a}(\mathrm{Rf})+(1-\mathrm{a}) \mathrm{RS}
$$


rentabilidad financiera tiene una ponderación igual a su proporción del costo del capital, mientras que la rentabilidad social tiene una participación equivalente al resto de la proporción que es necesaria para igualar la rentabilidad financiera al costo del capital. Es decir, se otorga a la rentabilidad social una participación hasta el límite que ésta permita igualar la rentabilidad compuesta al costo del capital. Dado que en el límite $\mathrm{R}=\mathrm{K}$ se deduce entonces que cuando en un proyecto $\mathrm{RF}$ es menor que $\mathrm{K}$, deberá existir una RS mínima para que el proyecto pueda ser considerado. En efecto, una simple manipulación algebráica demuestra que ese valor mínimo está dado por la siguiente expresión:

$$
\mathrm{RS}=\mathrm{K}-\mathrm{a}(\mathrm{RF}) / 1-\mathrm{a}
$$

Para el lector que se sienta incómodo con el razonamiento y las expresiones matemáticas, un sencillo ejemplo le permitiría comprender de manera más fácil el planteamiento anterior. Supóngase que en una institución cualquiera el costo del capital que forma su cartera de préstamos es de $10 \%$, y que además se somete un proyecto cuya rentabilidad financiera es de $8 \%$, pero que su rentabilidad social es de un $20 \%$. Con estos valores se puede calcular la rentabilidad compuesta de la siguiente forma:

$$
\mathrm{R}=0.8(8)+0.2(20)=10.4
$$

que es superior al costo del capital y por tanto el proyecto debe ser aceptado. Aún más, de acuerdo a la expresión para el cálculo de la RS mínima, se demuestra que para una RS por debajo de $16 \%$, el proyecto debe ser rechazado.

En este punto es muy seguro que el lector se esté preguntando que a cuál tasa de interés debe la institución financiar este proyecto. Obviamente, la máxima carga financiera que el proyecto soporta es $8 \%$, por encima de la cual dejará de ser "rentable" para el promotor. Es posible que en proyectos del tipo de microempresas, los beneficiarios otorguen más valor a la rentabilidad social que a la financiera, por lo que en nuestro ejemplo hipotético, el beneficiario esté dispuesto a aceptar un interés del $8 \% .^{5}$ Por otra parte, la institución aceptará este proyecto ya que su rentabilidad compuesta es mayor que el costo económico de los recursos que ésta utiliza, con lo cual deberá subsidiar en un $20 \%$ la tasa de interés de ese proyecto.

El segundo caso que se puede presentar es que la rentabilidad financiera sea mayor que el costo del capital, por lo que el coeficiente 
"a" será mayor que la unidad. En este caso, la expresión apropiada para el cálculo de la rentabilidad compuesta es la siguiente:

$$
\mathrm{R}=\mathrm{RF}(\mathrm{a}-1)+\mathrm{RS}(\mathrm{a})
$$

Se observa que la participación de la rentabilidad financiera es igual al exceso de ésta sobre el costo del capital, y que la participación de la rentabilidad social es equivalente al costo del capital más el porcentaje en que excede la rentabilidad financiera a dicho costo de capital. Al igual que en el caso anterior, en el límite $\mathrm{R}=\mathrm{K}$, por lo que en este caso, es también posible derivar una expresión para el valor mínimo que puede tomar RS:

$$
\mathrm{RS}=\mathrm{R}-\mathrm{RF}(\mathrm{a}-1) / \mathrm{a}
$$

Para fines de ilustración, es conveniente retomar de nuevo el caso del ejemplo anterior, pero esta vez asignando a RF un valor de $12 \%$ en vez de $8 \%$. En esta situación el valor de R será:

$$
\mathrm{R}=12(1.2-1)+20(1.2)=26.4 \%
$$

que excede con mucho al costo del capital de la institución. El valor mínimo que puede tomar RS para que el proyecto sea aceptado es $6.3 \%$, calculado sustituyendo en la expresión correspondiente.

De nuevo surge la inquietud sobre la tasa de interés que debería cobrar la institución en este caso. Obviamente que el límite que estaría dispuesto a aceptar los beneficiarios es el 12\%. Supóngase que éste es el valor. Si la institució . dedica su cartera a financiar ambos proyectos en proporciones iguales, estaría obteniendo unos ingresos suficientes para cubrir exactamente su costo de capital, por lo que en términos operativos podría decirse que estaría en su punto de equilibrio. ${ }^{6}$ No obstante, la rentabilidad compuesta total sobre la cartera es el $18.4 \%=$ $0.5(10.4)+0.5(26.4)$, que excede en un $84 \%$ a su costo de capital, lo cual es un indicador de la eficiencia de la institución o el programa en la consecución de sus objetivos.

La aplicación práctica del modelo anterior requiere de una medición del costo del capital para la institución y de un método para el cálculo de la rentabilidad social de cada proyecto. La primera tarea es sencilla, ya que para estos fines sólo es necesario un simple análisis de las fuentes de financiamiento. Para la evaluación social es recomendable que cada institución tenga su propio sistema, el cual puede 
construirse a partir de estudios elaborados para tales fines a costos razonables para cada institución, ya que los mismos partirían de criterios sencillos para la modificación de los precios de mercados.

Otro requerimiento quizás menos tangible o cuantitativo es el reconocimiento por parte de la institución de que una tasa de interés selectiva es un instrumento básico para la promoción de las pequeñas unidades productivas del sector informal.

\section{NOTAS}

1. La bibliografía en favor de una política selectiva de promoción del sector informal es extensa. Para una buena muestra de aquella que enfatiza en el financiamiento y la tasa de interés, véase, Carbonetto (1985), Mezera (187), Mizrahi (1987), Souza y Tokman (1987), y Tokman (1987).

2. El principal exponente de las políticas liberales para la promoción del sector informal es De Soto. Una presentación detallada de sus propuestas puede encontrarse en "El otro Sendero", Lima, Editorial el Barranco, 1986.

3. La literatura macroeconómica es bastante amplia en lo que se refiere a este aspecto. Sin embargo, algunas consideraciones desde el punto de vista del sector informal pueden encontrarse en Hann (1985) y Mezera (1987).

4. La no disponibilidad de financiamiento a largo plazo para alcanzar niveles aceptables de acumulación e incrementos de la productividad, y la imposibilidad de lograr dicho financiamiento por la vía del constreñimiento del consumo, es la principal razón por la cual las pequeñas unidades de producción del sector no se convierten en empresas modernas. En consecuencia, una política de promoción neutral, tendente a la liberalización no tiene ninguna posiblidad de lograr un aumento en los niveles de ingresos informales. Para una revisión detallada de éste y otros aspectos relacionados con la política de promoción, véase, Reyes (1989).

5. Se supone por ejemplo, que los salarios del propietario o cualquier otro familiar o promotor han sido deducidos de los ingresos para calcular los flujos de efectivo sobre los que a su vez se calcula la rentabilidad. 
6. Esto se deriva del hecho de que la tasa de interés promedio ponderada por la participación de cada proyecto es el $10 \%$, es decir, $0.5(0.08)+0.5(0.12)=0.1$.

\section{LITERATURA CITADA}

Carbonetto, D. "Políticas de Mejoramiento del Empleo en el Sector Informal Urbano (SIU)". En El Sector Informal Urbano en los Países Andinos. ILDIS-CEPESIU, Quito, 1985.

De Soto, H. El Otro Sendero. Lima, El Barranco, 1986.

Hann, H. El Sector Informal en Centroamérica. Investigaciones sobre empleo/27. PREALC, Santiago, 1985.

Mezera, J. Crédito y Capacitación para el Sector Informal. Investigaciones sobre empleo/29. PREALC, Santiago, 1987.

Mizrahi, R. Economía del Sector Informal: La Dinámica de las Pequeñas Unidades y su Viabilidad. Desarrollo Económico, 26, No. 104, 1987.

Reyes, R. El Sector Informal y la Microempresa en República Dominicana: Funcionamiento y Política de Promoción. Georgetown University, Washington, 1989. (en prensa).

Souza, P. y Víctor Tokman. Sector Informal. Funcionamientos y Políticas. Revista Internacional del Trabajo, 94, No. 3. OIT, Ginebra, 1976.

Tokman, V. Las Relaciones entre los Sectores Formal e Informal. Una Exploración sobre su Naturaleza. Revista de la CEPAL, Primer Semestre de 1978, CEPAL, Santiago, 1978. 